\title{
1 An integrative resource for investigating genetic model of drug response in
}

2 cancer

$3 \quad$ Yi Guo ${ }^{1}$, Xiang Chen ${ }^{2, *}$

$4{ }^{I}$ Department of Polymer Science and Engineering, Zhejiang University, Hangzhou, P.R. China, $5 \quad 310027$

$6{ }^{2}$ Institute of Pharmaceutical Biotechnology and the First Affiliated Hospital Department of Radiation 7 Oncology, Zhejiang University School of Medicine, Hangzhou 310058, P.R. China

8

$9 \quad$ E-mail:

10 Xiang Chen:xiang-chen@zju.edu.cn

11 *To whom correspondence should be addressed.E-mail:xiang-chen@zju.edu.cn

14 Abstract

\section{Background}

16 The most important goal of pharmacogenetics research is to obtain genetic models closely related to

17 drug efficacy in vivo and in vitro, which can be used to measure individual responses and then to 18 assess the sensitivity and toxicity of anticancer drugs. However, these models are scattered in 19 different pharmacogenetic studies and are difficult to be effectively used and analyzed.

\section{Results}


21 To this end, we have developed an integrated resource named iGMDR that provides the genetic

22 models of both clinical and preclinical anticancer drugs. iGMDR provides a unique resource for

23 mining the links between anticancer drugs and individual genomes, which will attempt to discover

24 cancer knowledge and unlock the potential value of this data.

\section{Conclusions}

26 We developed iGMDR as a web service whose code was released at GitHub repository of ModelLAB

27 (https://github.com/ModelLAB-ZJU/iGMDR), it also provided online access and application interface

28 (https://igmdr.modellab.cn). MySQL dump for the integrated resources was stored at zenodo.org

29 (DOI: 10.5281/ zencomod.1432561).

30 Keywords: Genetic model; Pharmacogenetics; Anticancer drug; Cancer

\section{BACKGROUND}

Pharmacogenetics (a portmanteau of pharmacology and genetics) is the study of the role of genetics in individual genetic characteristics $[1,2]$. It is one of the main bottlenecks in the implementation of personalized medicine due to the complexity of research [3, 4]. Traditionally, diseases have been treated largely based on an epigenetic understanding and a knowledge of population genetics of the disease, and results often fail in specific individual cases [5]. With the rapid development of geneticsrelated sequencing technology and the accumulation of knowledge due to various drug development, the relationship between the curative efficacy and the individual genetic characteristics has become increasingly clear, leading to more accurate prediction of the effects of treatment strategies based on

42 the genetic characteristics of patients, and thus we have obtained genetic models of some drugs with 43 widespread clinical application [3]. Cancer therapy is one of the challenging goals of precision 44 medicine and personalized medicine, and its occurrence is closely related to abnormal regulation of 
46 pharmacogenetics studies in cancer have been widely implemented and have produced many effective

47 genetic models that have been applied in clinical practice (NCCN guidelines, FDA drug labels) [7, 8].

48 Nevertheless, the pace of research in cancer pharmacogenetics has lagged far behind the need for

49 cancer precision medicine, and many cases of access to treatment remain based on traditional "one

50 treatment fits all" strategies [9]. More systematic studies and more effective data analysis are needed

51 if more individual-specific genetic models are to be obtained to improve the effectiveness of cancer

52 treatment. Experimental designs from in vitro to in vivo are an indispensable path for the application

53 of majority knowledge to clinical practice. Currently, there are several in vitro pharmacogenetics

54 studies based on cancer cell lines, such as CCLE [10], GDSC [11], CTRP [12], CGP [13], and MCLP

55 [14], and these studies have yielded many genetic models for specific drugs and specific types of

56 cancer. In addition, in vivo cancer studies of some model organisms have also yielded considerable

57 preclinical genetic models. While there are still many gaps and unpredictable challenges to translate

58 these genetic models into clinical practice, the value of these data should not be underestimated. To

59 this end, we constructed iGMDR to collect genetic models of anticancer drugs in different technical

60 systems and different research stages. Our research goal is to integrate big data content and create

61 new value based on these data.

\section{DATA CURATION AND ACCESSION}

64 Recently, iGMDR integrates datasets including ClinicalTrials, FDA, NCCN, ASCO

65 (https://www.asco.org), CCLE, GDSC, CTRP, MCLP, CGP, and literature on pharmacogenetic

66 studies. As described above, we collected genetic models of anticancer drugs from four sources: 1)

67 clinical guidelines, 2) approved drug labels, 3) scientific literature, and 4) experimental statistics (see

68 Figure 1, for detailed reference datasets on the online website). According to the identification stage,

69 it can be divided into clinical and preclinical models; According to the research object, it can be

70 divided into in vivo model and in vitro model; Genome, transcriptome, proteome model and logical

71 model (logical combination of events) were obtained according to the classification of the technical 
72 system. According to the model related event types mainly include SNV (simple nucleotide

73 variation), CNV (copy number variation), EXP (expression), SV (structural variation), SPV (splice

74 variant) and LN (cell lineage) [see Table S1 for more event types]. We standardized the data from

75 these sources, including tissue type, cancer type, drug, gene and model presentation (see Table S2 for

76 detailed process description). Eventually, we obtained about 154,100 models of 144 cancers of 30

77 tissue types, involving 1,040 different types of anticancer drugs (hormones, small molecules,

78 vaccines, antibody drugs, etc.). In order to build an efficient research resource of pharmacogenetics,

79 we integrated various information about drugs including chemical composition, structure, target,

80 signal pathway and classification, and information about genes includes functional description and

81 associated functional signaling pathways (see Table S3 for a list of detailed information sources).

\section{WEBSERVER CONSTRUCTION}

84 Based on the above integration process, we constructed an anticancer pharmacogenetics model

85 resource, iGMDR, whose code is open source and available on GitHub repository

86 (https://github.com/ModelLAB-ZJU/iGMDR) based on the GPLv3 license. The iGMDR online site

87 was built on an Apache HTTP server, all model data was stored in a MySQL database, and PHP was

88 used for backstage and front-end interactions (see Figure 1). Users can fully browse and download the

89 genetic models of anticancer drugs through different data types (cancer, tissue, drug, and gene). Based

90 on the intuitive understanding that pharmacogenetics researchers are usually interested in genetic

91 models involving specific genes and anticancer drugs, we also designed the relevant search and

92 profile interfaces, and the feedback information will be displayed visually. Specific drugs exhibitions

93 will include general information (composition, targets, signaling pathways, classification, and external

94 database ID), conformer structure (small molecule drugs), models of drug-related genes, the cancer

95 tissue distribution of drug-related models, the signaling pathways and function enrichment of drug-

96 related models, the drug-gene interaction network (the network was built through the number of drug-

97 related models), and the drug-related model list. Specific gene profiling page describes the basic 
98 information of the gene (gene summary, categories, and external database ID), gene-related signaling

99 pathways and functions, gene-associated anticancer drugs, the normal tissue distribution of gene

100 expression in human, the distribution of gene-related drug targets and signaling pathway, and gene-

101 drug interaction network (constructed by the number of gene-related models). These profiling and

102 data presentations will facilitate the understanding of specific anticancer drug and gene-related 103 genetic models.

\section{IMPLEMENTATION CASE}

Lung cancer is one of the most popular cancers in the world [15]. Over 7.15\% (COSMIC) of cancer patients have the defects (Point Mutations, Copy Number Variation, and Gene Expression) of STK11 gene. STK11 is a tumor suppressor that functions in the regulation of cell polarity, TP53 activity, and cell-cycle arrest. In our integrated resources, we can find that the most influential cancer tissue related to STK11 is Lung. For Lung cancers harboring STK11 mutations, we can predict the intervention effect of the corresponding 113 drugs. Of course, the genome variation of cancer patients is complex and diverse, and the genetic indicator from a single model may not be able to predict the final efficacy of drugs, so our resources also provide other indicators of genetic variation to predict the intervention effect of these 113 drugs.

Mahoney et al. have reported that non-small cell lung cancer (NSCLC) cell lines with mutations of STK11 and KRAS genes are sensitive to MEK inhibitors showing a dose-dependent inhibition of growth rate, while when the two are mutated alone that will show opposite inhibition [16]. In addition, several groups of clinical trials (e.g. PF-4554878, VS-6063) recruiting cancer patients for NSCLC have been recommended to test the status of STK11 [17, 18]. As shown in Figure 2, we constructed a gene-related model network of STK11 in our resources, including all the genetic indicators of these 113 drugs (red dots). Importantly, these drug interventions for NSCLC can be 
The essence of tumorigenesis has been widely believed to be the result of aberrant cell signal transduction caused by genetic variation that further disrupt these signaling pathways as cancer progresses, and this process will become much more complicated than that of tumorigenesis [19]. Therefore, the traditional targeted therapy for a single target is often less effective for cancer treatment. Through the query of our resources, multiple targeted drugs are sensitive in terms of Lung cancer with STK11 gene defects, and the genes targeted by these therapeutic drugs participate in different signaling pathways (see Table S4). In addition, the predictive genes involved in these drugrelated treatment models are also involved in these different signaling pathways (Figure 2). Based on this, we can design new treatment schemes to deal with the same or different pathways with abnormal signal transduction to improve the therapeutic effect of cancer. In other words, we can use this network to further infer new pharmaceutical strategies.

Interestingly, some kind of strategy at the signaling pathway level and important inferences for cancer treatment had been really reflected in the model networks that we built on this resource. STK11 is frequently mutated in NSCLC that acts as a tumor suppressor by activating AMPK (5' AMP-activated protein kinase) signaling, whereas loss of STK11 by point mutation or deletion suppresses AMPK, leading to increased mTOR signaling. Recently researchers have investigated the effects of STK11 mutation and mTOR inhibition on cell signaling pathways in NSCLC cell lines, suggesting a feedback mechanism that STK11 mutant cell lines increased insulin-like growth factor receptor (IGFR) activity, and inhibition of the IGFR activity was shown to downregulate the mTOR pathway[20]. The results supported the investigation of IGFR inhibitors in combination with drugs targeting the mTOR pathway, particularly for tumors bearing STK11 alterations [21].

By dephosphorylation of PIP3, PTEN negatively regulates PI3K/AKT leading to tumor suppression in NSCLC [22]. PTEN inactivation/loss therefore increases activity of the PI3K-AKTmTOR pathway and further suggest that NSCLC patients might afford an opportunity for exploitation of anti-PI3K-AKT/mTOR-targeted therapies. Several studies have shown this subset of lung cancers treated with PI3K/AKT inhibitors combining with mTOR inhibitors (rapamycin) [16]. Until now, targeting of TP53 signaling has proved to be highly disappointing. However, recent studies indicate 
151 abundant crosstalk between TP53 and the AKT/mTORC1 signaling pathway can determine the choice

152 of response to TP53. These results could have important pharmacological consequences for the 153 potential development of PI3K/Akt inhibitors against TP53-deficient cancers [23, 24].

154 To sum up, the integrated model information will provide guidance for personalized treatment of 155 cancer patients, and users can also infer new therapeutic strategies by connecting the model with the 156 signal transduction network so as to improve the defects of single-targeted treatment (see Figure 3).

157 These directions may help to identify patients who would most benefit from alternative single or dual 158 pathway inhibition potentially leading to a revision in current molecular testing guidelines, and also 159 demonstrate the advantages of integrating resources.

\section{SUMMARY AND APPLICATION DIRECTIONS}

Drug genetics model can effectively achieve precision anticancer treatment $[25,26]$. To release the value of existing data, we have established a draft principle to collect the drug genetics model.

iGMDR is the first integrated data resource to provide a predictive model for the therapeutic effect of anticancer drugs. Compared with the resources we have integrated, the current resources only provide a single genetics model of anticancer drug ("experimental statistics" for CCLE and GDSC or "clinical guidelines" for NCCN guidelines and FDA drug labels), and the data volume is also different by orders of magnitude, which greatly limits the application value of such data. The interactive design and data visualization of the iGMDR website provides both macro and micro insights for pharmacogenetics researchers in the cancer drug genetics model. For example, we can design a new sequencing panel for cancer drug efficacy prediction; We can link drug-related targets and signaling pathways to design combinatorial strategies for cancer therapy. In conclusion, iGMDR will continue to integrate relevant pharmacogenetic studies into cancer clinical and research therapy. 
176 The authors declare that they have no competing interests.

177

178

179

180

181

182

183

184

185

186

187

188

189

References

190 1. Pirmohamed M: Pharmacogenetics: past, present and future. Drug Discov Today 2011, 16(19-20):852-861.

192 2. Pirmohamed M: Pharmacogenetics and pharmacogenomics. Br J Clin Pharmacol 2001, $193 \quad$ 52(4):345-347.

194 3. Scott SA: Personalizing medicine with clinical pharmacogenetics. Genet Med 2011, 195 13(12):987-995. 
196 4. Hamburg MA, Collins FS: The path to personalized medicine. $N$ Engl J Med 2010,

$197 \quad \mathbf{3 6 3}(4): 301-304$.

198 5. Collins FS, Varmus H: A new initiative on precision medicine. $N$ Engl J Med 2015, $199 \quad 372(9): 793-795$.

6. Chambliss AB, Chan DW: Precision medicine: from pharmacogenomics to pharmacoproteomics. Clin Proteomics 2016, 13:25.

202

7. Chakravarty D, Gao J, Phillips SM, Kundra R, Zhang H, Wang J, Rudolph JE, Yaeger R,

8. Relling MV, Dervieux T: Pharmacogenetics and cancer therapy. Nat Rev Cancer 2001,

9. Dancey JE, Bedard PL, Onetto N, Hudson TJ: The genetic basis for cancer treatment decisions. Cell 2012, 148(3):409-420.

10. Barretina J, Caponigro G, Stransky N, Venkatesan K, Margolin AA, Kim S, Wilson CJ, Lehar 
218 13. Garnett MJ, Edelman EJ, Heidorn SJ, Greenman CD, Dastur A, Lau KW, Greninger P, 219 Thompson IR, Luo X, Soares J et al: Systematic identification of genomic markers of drug sensitivity in cancer cells. Nature 2012, 483(7391):570-575.

14. Li J, Zhao W, Akbani R, Liu W, Ju Z, Ling S, Vellano CP, Roebuck P, Yu Q, Eterovic AK et al: Characterization of Human Cancer Cell Lines by Reverse-phase Protein Arrays. Cancer Cell 2017, 31(2):225-239.

15. Rafiemanesh H, Mehtarpour M, Khani F, Hesami SM, Shamlou R, Towhidi F, Salehiniya H, Makhsosi BR, Moini A: Epidemiology, incidence and mortality of lung cancer and their

16. Mahoney CL, Choudhury B, Davies H, Edkins S, Greenman C, van Haaften G, Mironenko T, Santarius T, Stevens C, Stratton MR et al: LKB1/KRAS mutant lung cancers constitute a genetic subset of NSCLC with increased sensitivity to MAPK and mTOR signalling inhibition. British Journal of Cancer 2009, 100(2):370-375.

17. Mohseni M, Sun J, Lau A, Curtis S, Goldsmith J, Fox VL, Wei C, Frazier M, Samson O, Wong KK et al: A genetic screen identifies an LKB1-MARK signalling axis controlling the Hippo-YAP pathway. Nat Cell Biol 2014, 16(1):108-117.

18. Nguyen HB, Babcock JT, Wells CD, Quilliam LA: LKB1 tumor suppressor regulates AMP kinase/mTOR-independent cell growth and proliferation via the phosphorylation of Yap. Oncogene 2013, 32(35):4100-4109.

19. Drier Y, Sheffer M, Domany E: Pathway-based personalized analysis of cancer. Proc Natl Acad Sci U S A 2013, 110(16):6388-6393. 
242 21. Yilmaz E, Byers LA, Diao LX, Giri U, Gudikote J, Fan YH, Wang J, Coombes KR, Girard L, 243 Minna JD et al: Use of proteomic analysis of LKB1/AMPK/mTOR pathways to identify IGF-1R pathway upregulation with LKB1 loss or mTOR inhibition in NSCLC: Implications for targeted combinations. Journal of Clinical Oncology 2012, 30(15).

22. Davidson L, Maccario H, Perera NM, Yang X, Spinelli L, Tibarewal P, Glancy B, Gray A, Weijer CJ, Downes CP et al: Suppression of cellular proliferation and invasion by the concerted lipid and protein phosphatase activities of PTEN. Oncogene 2010, 29(5):687697.

23. Skladanowski A, Bozko P, Sabisz M, Larsen AK: Dual inhibition of PI3K/Akt signaling and the DNA damage checkpoint in p53-deficient cells with strong survival signaling -

24. Duan L, Maki CG: The IGF-1R/AKT pathway determines cell fate in response to p53.

25. Ma Q, Lu AY: Pharmacogenetics, pharmacogenomics, and individualized medicine.

26. Huang L, Fernandes H, Zia H, Tavassoli P, Rennert H, Pisapia D, Imielinski M, Sboner A, Rubin MA, Kluk M et al: The cancer precision medicine knowledge base for structured 
bioRxiv preprint doi: https://doi.org/10.1101/555789; this version posted November 15,2019 . The copyright holder for this preprint (which was not certified by peer review) is the author/funder, who has granted bioRxiv a license to display the preprint in perpetuity. It is made available under aCC-BY-NC-ND 4.0 International license.

\begin{tabular}{|c|c|c|c|c|c|c|c|}
\hline Clinical guidelines & \multirow[t]{5}{*}{ Gene } & Event & Cancer type & \multirow{2}{*}{$\begin{array}{l}\text { Drug } \\
\text { EGFR inhibitors }\end{array}$} & & \multicolumn{2}{|r|}{ iGMDR } \\
\hline Approved drug labels & & & Lung cancer & & & & Table \\
\hline Scientific literature & & erM & & Nilotinih & $\rightarrow$ & & row 1 \\
\hline Experimental statistics & & Amp & & Cabozantinib & & & now 3 \\
\hline Data Sources & & & & & & & Models Curation \\
\hline
\end{tabular}

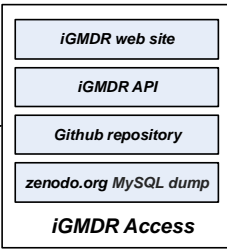


283 Figure 1. The schematic overview of iGMDR. The first column describes the data sources of the

284 genetic model of anticancer drugs. The second column describes the data integration process. The

285 third column is how the data is accessed and retrieved. 


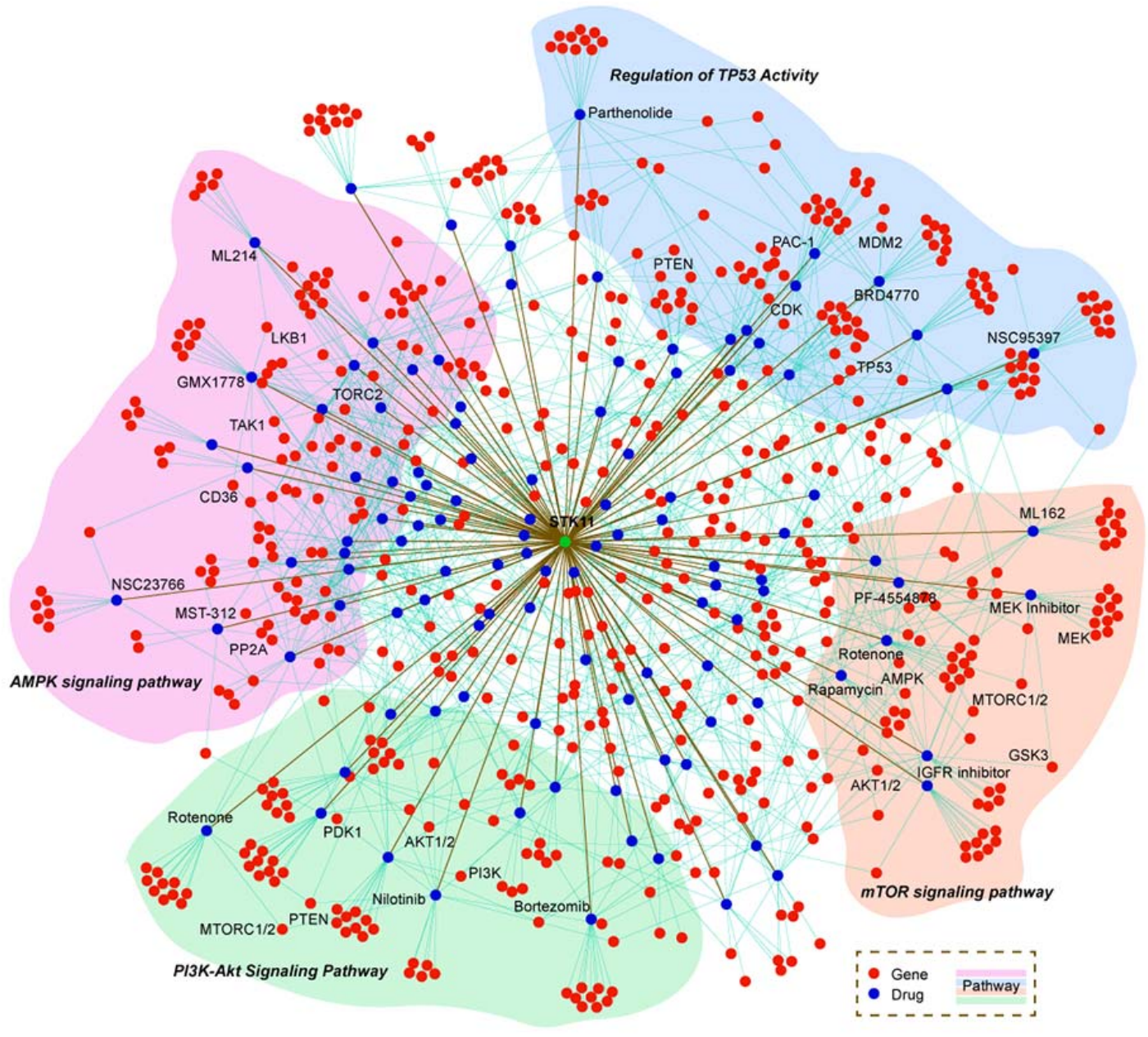

311 Figure 2. STK11 gene related drug model (drug-gene) network. Model related drugs and genes

312 were connected by straight lines, while drugs directly related to STK11 genes were connected by significantly thick lines. The background color blocks represent the signaling pathways of the genes associated with these models. 


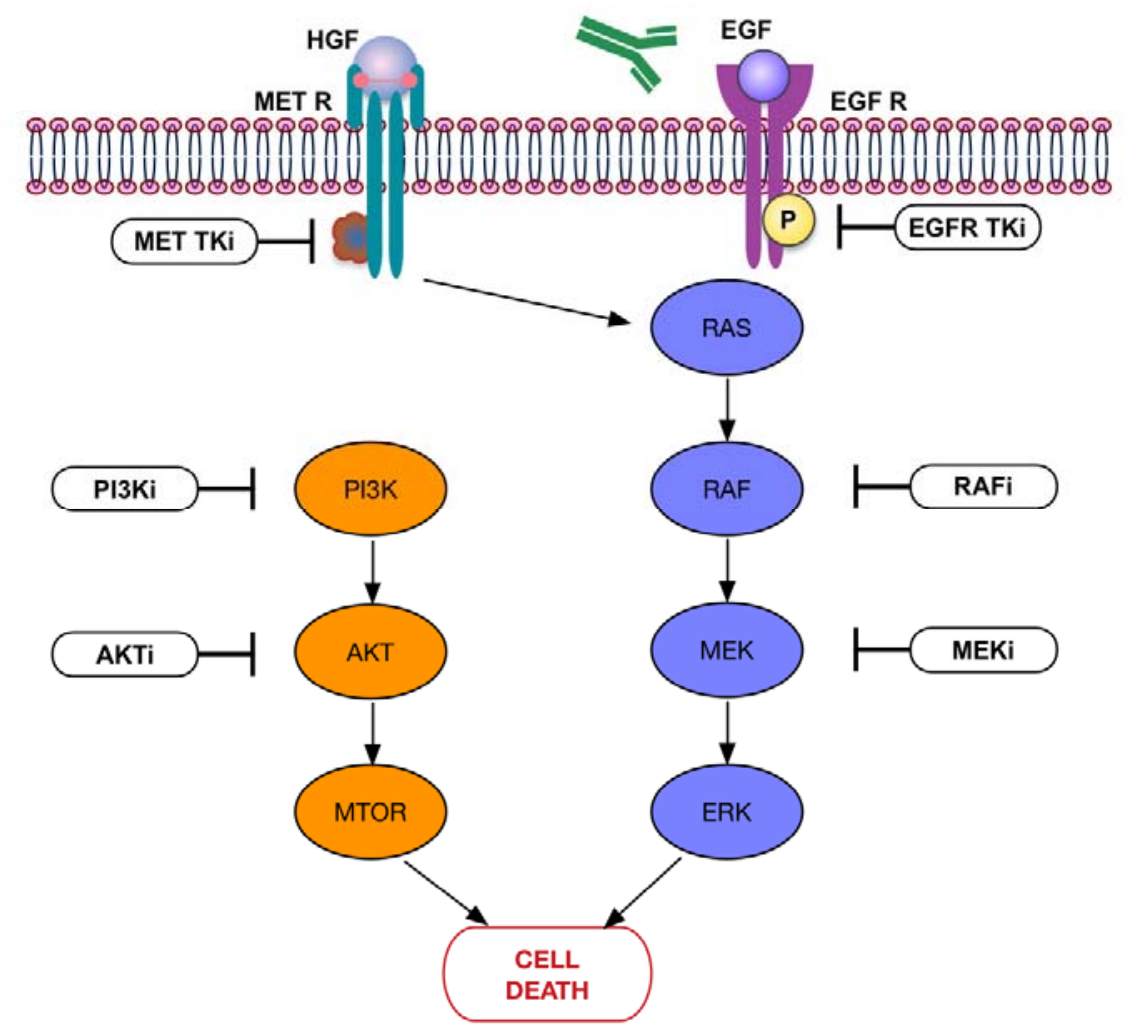

323 Figure 3. Mechanism diagram of cancer treatment strategy based on cellular signaling

324 pathways. This diagram simulates the processes of drug intervention against abnormal signaling pathways in cancer cells. 


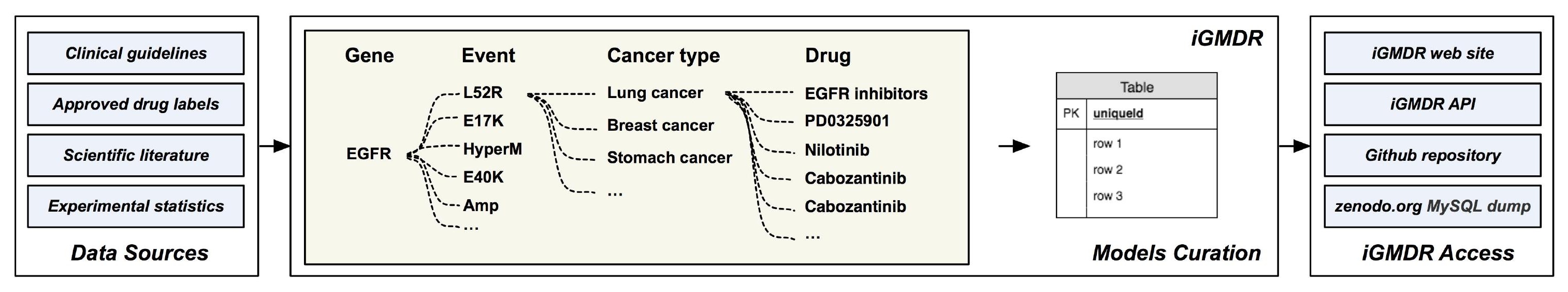




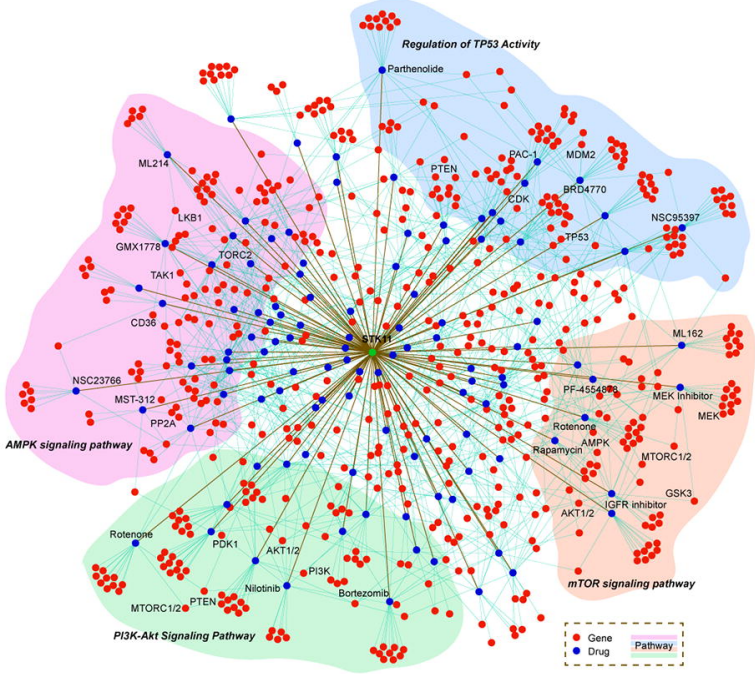




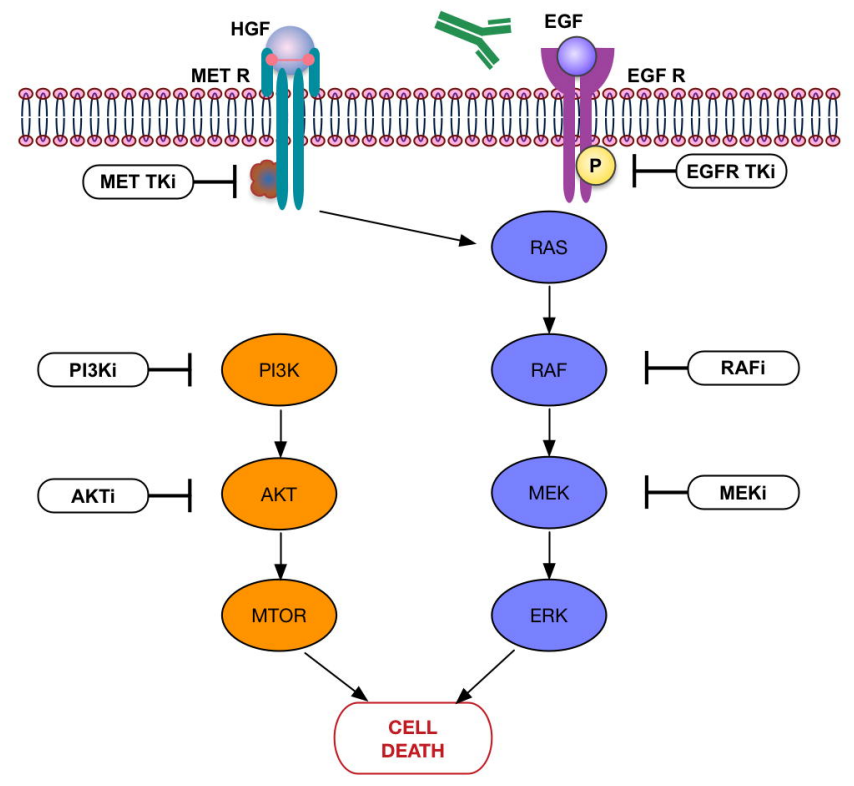

